\title{
Typ-2-Diabetiker profitieren kardiovaskulär von frühem Gewichtsverlust
}

Hintergrund und Fragestellung: Bekanntermaßen führt ein Gewichtsverlust zur Abnahme kardiovaskulärer Risikofaktoren. Die Ergebnisse der Look-AHEAD-Studie zeigten jedoch keine Reduktion kardiovaskulärer Ereignisse im Rahmen einer intensiven Lebensstil-Intervention bei Typ-2-Diabetikern. In dieser Post-Hoc-Analyse der Look-AHEAD-Daten wurde daher die Frage adressiert, ob das Ausmaß des Gewichtsverlustes beziehungsweise die Änderung der körperlichen Fitness einen Effekt auf das Auftreten kardiovaskulärer Ereignisse hat.

Patienten und Methoden: Es wurden die Daten der LookAHEAD-Studie (RCT mit 5154 übergewichtigen oder adipösen Typ-2-Diabetikern im Alter zwischen 45 und 76 Jahren, Vergleich herkömmliche Diabe-

Originalie

The Action for Health in Diabetes (Look AHEAD) Study Group. Association of the magnitude of weight loss and changes in physical fitness with long-term cardiovascular disease outcomes in overweight or obese people with type 2 diabetes: a post-hoc analysis of the Look AHEAD randomised clinical trial.

Lancet Diabetes Endocrinol.

2016 Nov;4(11):913-21. tesversorgung vs. intensivierter Lebensstilintervention) hinsichtlich der Frage betrachtet, ob die Gewichtsreduktion und die Veränderung der körperlichen Fitness (Metabolische ÄquivalenteMETs) im ersten Jahr der multimodalen Lebensstil-Intervention mit den Endpunkten assoziiert sind. Der primäre Endpunkt bestand aus kardiovaskulärem Tod, nicht tödlichem Herzinfarkt, nicht tödlichem Schlaganfall oder Klinikeinweisung aufgrund von Angina Pectoris. Der sekundäre Endpunkt schloss zusätzlich Gesamtmortalität, pAVK, Koronarinterventionen/Bypass und Hospitalisierung wegen Herzinsuffizienz mit ein.

Ergebnisse: Betrachtet man die Gesamt-Studienpopulation so hatten Individuen mit einem Gewichtsverlust von mind. 10\% im ersten Jahr ein 21\% niedrigeres Risiko, den primären Endpunkt zu erleiden (HR 0,79; 95\%-KI 0,64-0,98; p=0,034) und eine 24\%ige Reduktion bzgl. des sekundären Endpunktes (HR 0,76; 95\%-KI 0,63-0,91; p=0,003) im Vergleich zur Gruppe mit stabilem Gewicht oder Gewichtszunahme. Ein Anstieg der körperlichen Fitness um mind. 2 METs war mit einer signifikanten Reduktion des sekundären Endpunkts (HR 0,77; 95\%-KI 0,61 -0,96; $\mathrm{p}=0,023)$ verbunden, keinen Effekt gab es auf den primären Endpunkt $(\mathrm{p}=0,079)$. Beim Vergleich Kontroll- zu Interventionsgruppe hatten die Interventions-Teilnehmer mit $>10 \%$ Gewichtsverlust auch ein niedrigeres Risiko für den primären (-21\%) und sekundären Endpunkt (-20\%), die Verbesserung der körperlichen Fitness hatte aber keinen Effekt auf Ereignisraten.

Schlussfolgerungen: Trotz der Negativ-Ergebnisse in der Gesamtstudie besteht eine Assoziation zwischen der Gewichtsreduktion im ersten Jahr und den kardiovaskulären Endpunkten im Langzeitverlauf bei Typ-2-Diabetes. Das, so die Autoren, unterstreicht erneut die Dringlichkeit zur Entwicklung von effektiven Strategien im Bereich der Lebensstilintervention, die eine stärkere Gewichtsreduktion und deren Erhalt ermöglichen.

\section{- Kommentar von PD Dr. med. Knut Mai}

\section{Gewichtsreduktion lohnt sich kardiovaskulär wohl doch}

Trotz aller Limitation dieser Post-Hoc-Analyse führen auch diese Daten zu interessanten Schlussfolgerungen. So sind die Ergebnisse einer Lebensstil-Modifikationen mit dem Ziel der Gewichtsreduktion und Steigerung der körperlichen Fitness bei Patienten mit Typ-2-Diabetikern doch möglicherweise nicht ganz so enttäuschend, wie dies initial nach Veröffentlichung der primären Ergebnisse von Look AHEAD angenommen wurde. So scheint besonders das Ausmaß der Gewichtsreduktion einen wesentlichen Effekt auf die Langzeitergebnisse zu haben. Dies macht ja auch im Kontext der positiven Effekte der bariatrischen Chirurgie auf die Mortalität Sinn, denn die erreichte Gewichtsreduktion ist hier ja deutlich stärker als dies momentan durch Lebensstil-Interventionen erreicht werden kann. Bekanntermaßen ist aber vor allem, und das wird verständlicherweise auch mehrfach von den Autoren adressiert, der Erhalt einer Gewichtsreduktion nach Intervention momentan eine der schwierigsten Herausforderungen. Inwieweit In- terventionen über Jahre (insofern dies populationsbezogen überhaupt umgesetzt werden kann) einen Effekt haben oder ob neuartige Strategien identifiziert werden müssen und können, ist momentan unklar. Hier sind daher auch weiterhin umfassende Anstrengungen nötig.

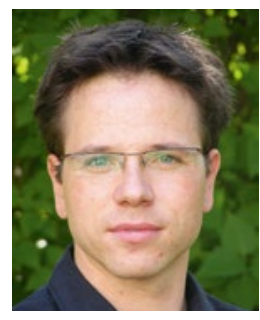

PD Dr. med. Knut Mai

Medizinische Klinik m.S. Endokrinologie,

Diabetes und Ernährungsmedizin

Charité - Universitätsmedizin Berlin

Campus Mitte, Charitéplatz 1, 10117 Berlin

Knut.Mai@charite.de 